Supplement of Biogeosciences, 14, 257-269, 2017

http://www.biogeosciences.net/14/257/2017/

doi:10.5194/bg-14-257-2017-supplement

(C) Author(s) 2017. CC Attribution 3.0 License.

(c) (i)

Supplement of

\title{
Species-specific temporal variation in photosynthesis as a moderator of peatland carbon sequestration
}

\author{
Aino Korrensalo et al. \\ Correspondence to: Aino Korrensalo (aino.korrensalo@uef.fi)
}

The copyright of individual parts of the supplement might differ from the CC-BY 3.0 licence. 
Table S1. Parameter estimates and standard errors of month- and species-wise hyperbolic light response models and the random effects of the models. Sample $\mathrm{n}$ is denoted with an asterisk (*), when light response parameters are calculated from a combined sample from the previous and following month.

\begin{tabular}{|c|c|c|c|c|c|c|c|c|c|c|c|c|c|c|c|}
\hline \multirow[b]{2}{*}{ Species } & \multirow[b]{2}{*}{ Month } & \multirow[b]{2}{*}{$\mathrm{n}$} & \multicolumn{6}{|l|}{ Fixed part } & \multicolumn{7}{|c|}{ Random part } \\
\hline & & & $\mathrm{k}$ & SE & Pmax & $\mathrm{SE}$ & $\mathrm{R}$ & $\mathrm{SE}$ & $\begin{array}{l}\text { SD } \\
(\text { Pmax })\end{array}$ & $\begin{array}{l}\text { SD } \\
(k)\end{array}$ & $\begin{array}{l}\text { SD } \\
\text { (R) }\end{array}$ & $\begin{array}{l}\text { SD } \\
\text { (residual) }\end{array}$ & $\begin{array}{l}\text { Corr } \\
\text { (Pmax,k) }\end{array}$ & $\begin{array}{l}\text { Corr } \\
(\text { Pmax,R) }\end{array}$ & $\begin{array}{l}\text { Corr } \\
(\mathrm{k}, \mathrm{R})\end{array}$ \\
\hline \multirow{5}{*}{ Sphagnum fuscum } & May & 4 & 138.4 & 7.0 & 710.4 & 75.1 & -125.1 & 9.6 & 130.0 & 12.1 & 16.6 & 0.7 & 0.8 & 0.2 & -0.4 \\
\hline & June & $6^{*}$ & 130.6 & 7.6 & 617.7 & 76.1 & -146.8 & 16.2 & 169.9 & 12.6 & 35.6 & 6.3 & 1.0 & 0.7 & 0.7 \\
\hline & July & 2 & 114.6 & 11.0 & 432.4 & 39.5 & -190.5 & 19.6 & 38.7 & & 18.8 & 5.4 & & -1.0 & \\
\hline & August & 4 & 96.8 & 43.1 & 423.3 & 53.4 & -230.1 & 26.4 & 63.8 & & & 45.0 & & & \\
\hline & September & 4 & 117.1 & 13.6 & 390.1 & 52.1 & -201.0 & 26.0 & 90.2 & 23.3 & 45.1 & 1.3 & 0.5 & 1.0 & 0.2 \\
\hline \multirow{5}{*}{ S. angustifolium } & May & 4 & 206.5 & 32.0 & 807.0 & 95.2 & -207.7 & 20.8 & 164.9 & 55.4 & 36.0 & 0.1 & 0.8 & 0.6 & 0.7 \\
\hline & June & $7 *$ & 194.3 & 19.4 & 649.5 & 95.5 & -167.4 & 27.6 & 233.9 & 47.6 & 67.6 & 0.1 & 0.7 & -0.5 & 0.1 \\
\hline & July & 3 & 155.6 & 129.9 & 436.9 & 87.7 & -117.9 & 39.2 & 93.7 & & & 56.0 & & & \\
\hline & August & 6 & 217.3 & 9.6 & 872.6 & 40.9 & -416.2 & 39.7 & 90.5 & & 8.0 & 6.2 & & -0.5 & \\
\hline & September & 3 & 262.3 & 253.4 & 688.5 & 105.5 & -215.7 & 38.6 & 92.5 & & & 53.2 & & & \\
\hline \multirow{5}{*}{ S. balticum } & May & 2 & 171.4 & 85.1 & 386.8 & 78.6 & -144.2 & 52.0 & 73.2 & & 48.3 & 19.5 & & -1.0 & \\
\hline & June & $5^{*}$ & 150.0 & 24.3 & 391.1 & 40.7 & -155.7 & 29.0 & 77.6 & 29.5 & 54.5 & 13.5 & -1.0 & -1.0 & 1.0 \\
\hline & July & 3 & 113.2 & 4.7 & 395.4 & 61.3 & -167.5 & 36.5 & 83.9 & & 49.8 & 2.4 & & -1.0 & \\
\hline & August & 3 & 234.3 & 17.9 & 632.8 & 87.2 & -323.4 & 27.2 & 118.5 & & 36.9 & 4.9 & & 1.0 & \\
\hline & September & 5 & 84.1 & 13.9 & 346.7 & 55.5 & -183.4 & 26.1 & 108.2 & 1.0 & 50.0 & 13.7 & 0.1 & 0.1 & 1.0 \\
\hline \multirow{2}{*}{ S. magellanicum } & May & 2 & 117.4 & 57.4 & 576.1 & 126.7 & -175.1 & 37.5 & 161.6 & & & 54.0 & & & \\
\hline & June & $5^{*}$ & 203.1 & 37.1 & 611.3 & 57.3 & -172.9 & 17.4 & 132.5 & 81.2 & 40.9 & 11.3 & -0.1 & 0.6 & -0.9 \\
\hline
\end{tabular}




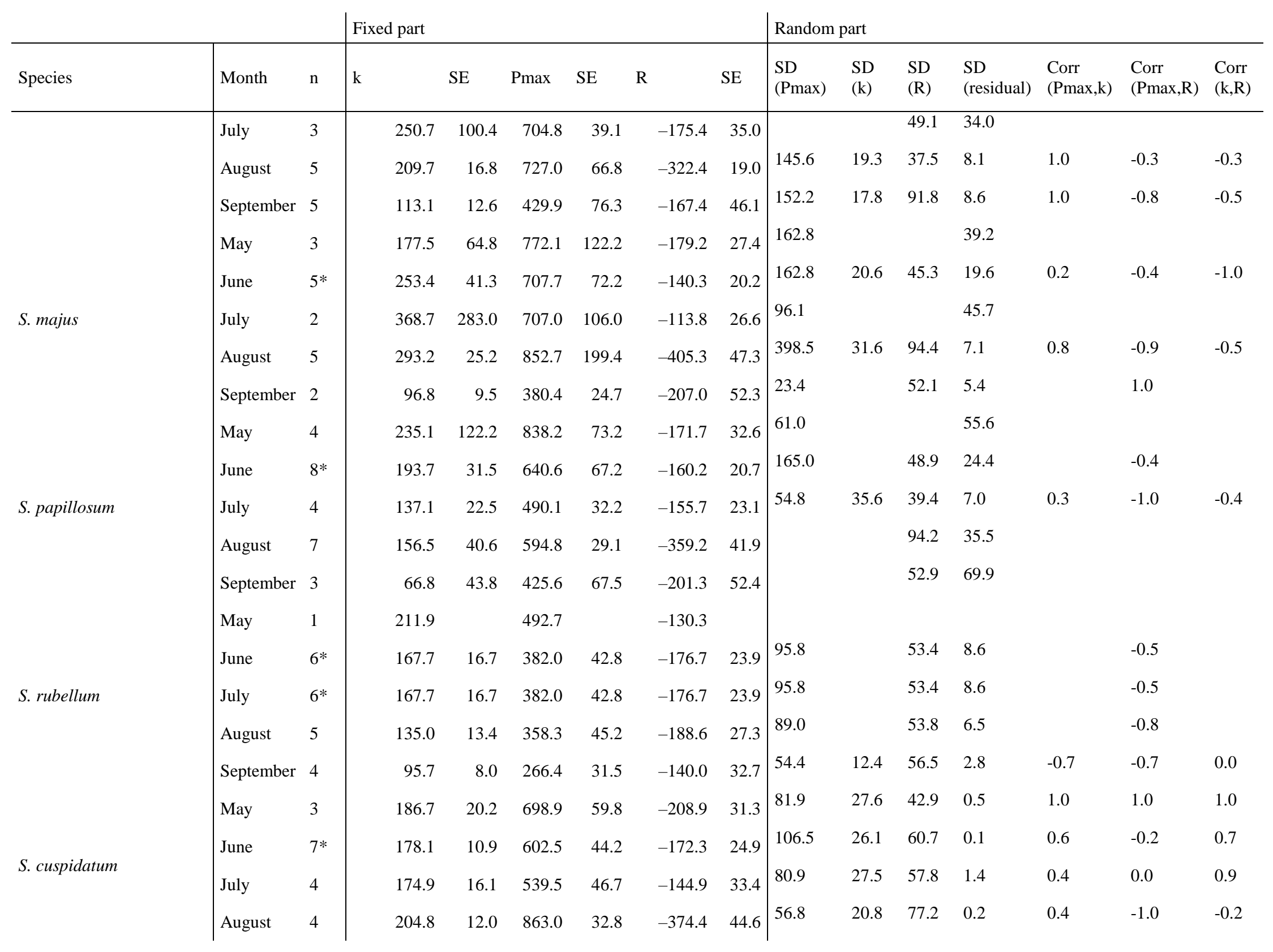




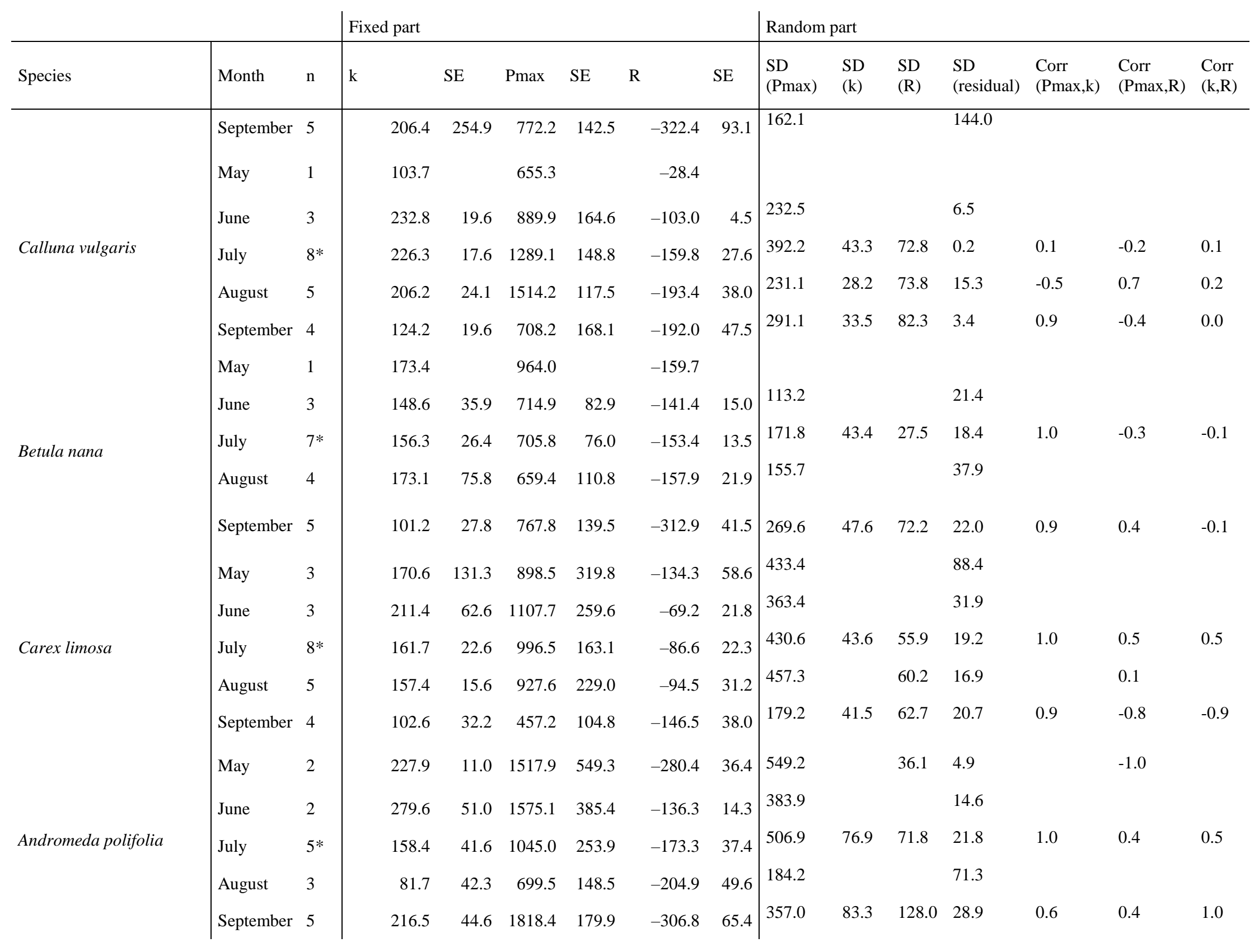




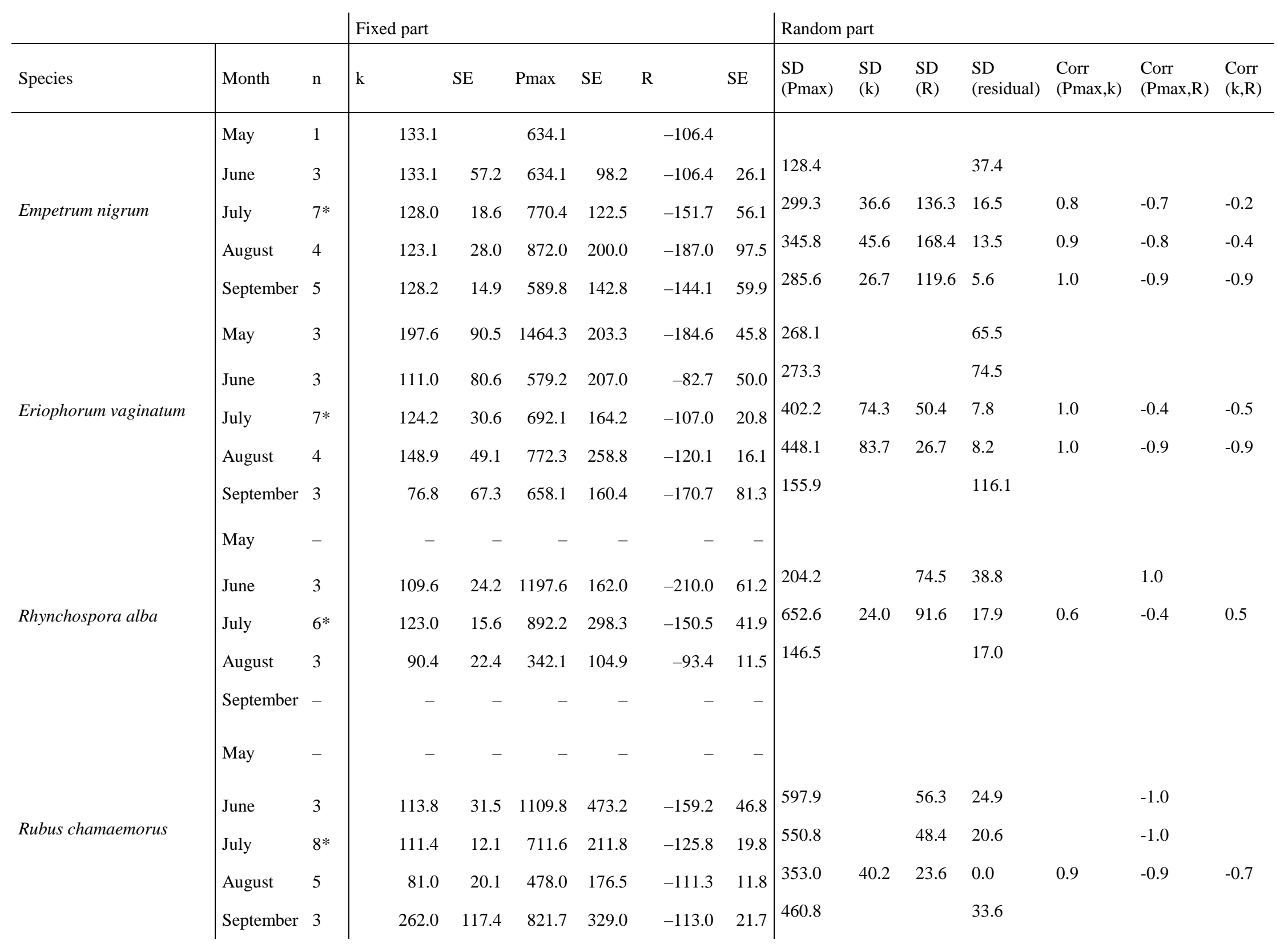




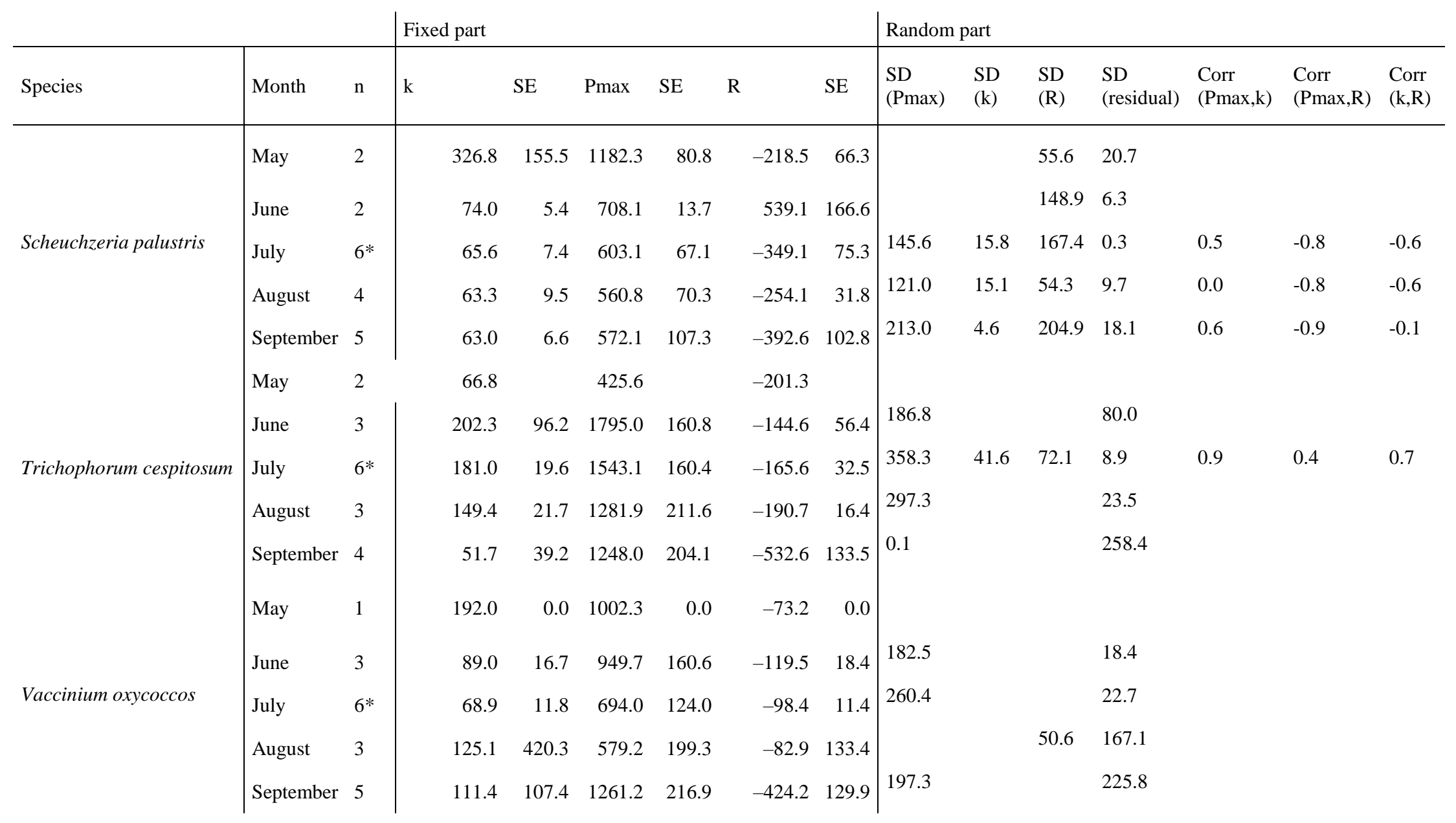


Table S2. Species-specific conversion factors (b1) to convert vascular plant cover (\%) into LAI ( $\mathrm{m}^{2}$ $\mathrm{m}^{-2}$ ) and the $\mathrm{R}^{2}$-values of liner regression $(\mathrm{LAI}=\mathrm{b} 1 *$ cover $)$.

\begin{tabular}{lll} 
Species & $\mathrm{b} 1$ & $\mathrm{R}^{2}$ \\
\hline C. vulgaris & 0.013 & 0.58 \\
B. nana & 0.009 & 0.79 \\
C. limosa & 0.017 & 0.61 \\
A. polifolia & 0.008 & 0.70 \\
E. nigrum & 0.009 & 0.87 \\
E. vaginatum & 0.013 & 0.75 \\
R. alba & 0.015 & 0.71 \\
R. chamaemorus & 0.011 & 0.61 \\
S. palustris & 0.021 & 0.62 \\
T. cespitosum & 0.021 & 0.27 \\
V. oxycoccos & 0.011 & 0.90 \\
\hline
\end{tabular}


Figure S3. A photograph of the study site.

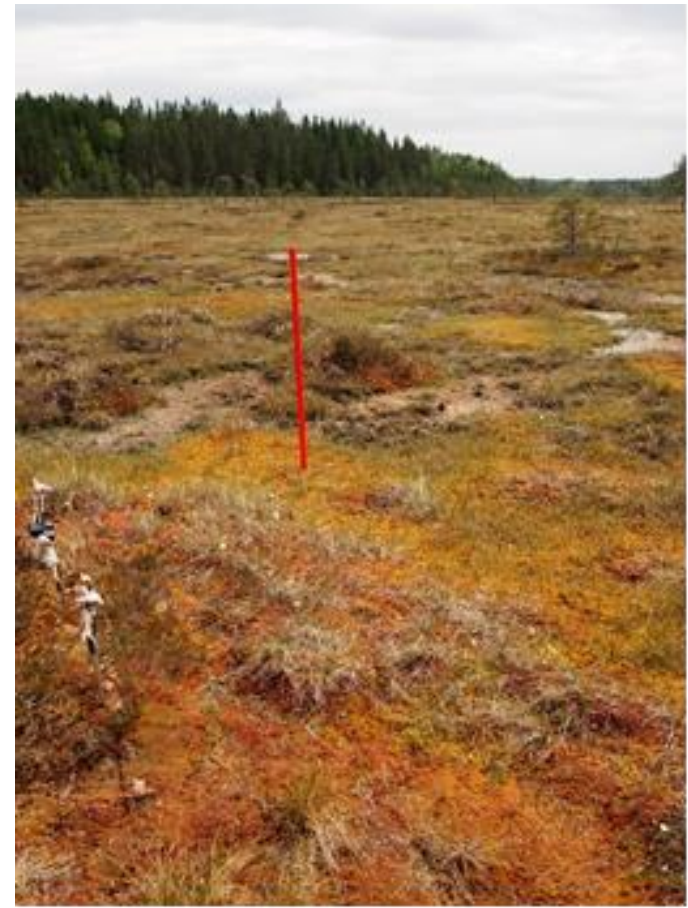

